\title{
Utilidad de los biomarcadores en insuficiencia cardiaca en la práctica clínica
}

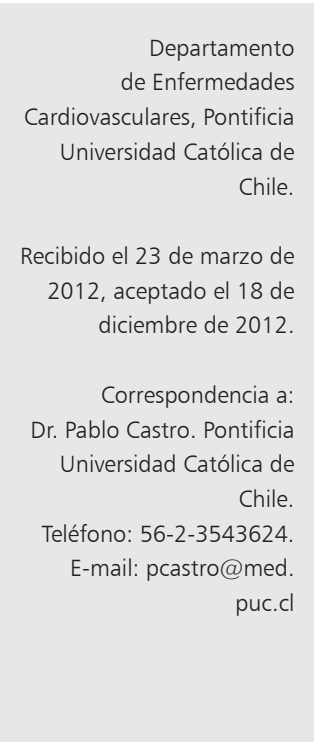

\author{
ALEJANDRO PAREDES C., JULIÁN VEGA, ANA DE LEÓN, \\ ANDRÉS KANACRI, PABLO CASTRO, RICARDO BAEZA
}

\section{Clinical usefulness of biomarkers in cardiac failure}

The assessment of patients with a suspected cardiac failure aims to an early and precise diagnosis and risk stratification. Only natriuretic peptides have demonstrated to be clinically useful. Brain natriuretic peptide stands out due to its diagnostic and prognostic value. However its results should be cautiously interpreted in the clinical context, bearing in mind possible confounders. The combination of markers can provide a better risk stratification and compensates the limitations of individual markers. Each new marker gives a new insight on the underlying physiopathology of cardiac failure and proposes new therapeutic approaches.

(Rev Med Chile 2013; 141: 1560-1569)

Key words: Biological markers; Heart failure; Prognosis; Troponin.
L a insuficiencia cardiaca (IC) es una condición altamente prevalente, presentando ciertas limitaciones en el diagnóstico, tratamiento y estratificación pronóstica. Sigue siendo una de las causas más importantes de hospitalización, morbilidad y mortalidad a nivel mundial ${ }^{1}$.

Se estima que 5 millones de personas en Estados Unidos de Norteamérica son portadores de IC, y que aproximadamente 550.000 casos nuevos son diagnosticados cada año. Las hospitalizaciones por este motivo han aumentado de 402.000 en 1979 a 1.101 .000 en 2004 (National Hospital Discharge Survey), y el costo anual se estima en 56 billones de dólares, 70\% de los cuales se debe a hospitalizaciones ${ }^{2}$.

Muchos pacientes con disnea aguda tienen múltiples trastornos médicos coexistentes que pueden complicar el enfrentamiento clínico. La incertidumbre en el diagnóstico provoca una mayor estadía hospitalaria, costos en salud y peor pronóstico, aumentando las posibilidades de reingreso o incluso muerte ${ }^{3}$.
La IC es vista como un fenómeno complejo resultante de alteraciones locales a nivel del cardiomiocito/intersticio y alteraciones sistémicas resultantes de respuestas compensadoras desadaptativas (activación simpática y neurohumoral) o secundarias a la incapacidad del miocardio de suplir las demandas metabólicas de los tejidos (estrés oxidativo e inflamación) ${ }^{4}$.

Existe un conjunto cada vez más amplio de sustancias bioquímicas circulantes que reflejan distintos aspectos de la fisiopatología de la IC, son los llamados biomarcadores.

En la práctica clínica, pueden ser usados para diagnosticar un problema médico, como herramienta en la estratificación de enfermedad o un indicador de diagnóstico diferencial.

El presente artículo se centra en la utilidad en la práctica clínica habitual de los marcadores bioquímicos de la IC, tratando de destacar sus debilidades y fortalezas, poniendo hincapié en aquellos con mayor evidencia científica demostrada a la fecha con proyección clínica. 


\section{Definición}

En el año 2001, el National Institutes of Health (NIH), estableció la definición de biomarcador como una característica objetivamente medida y evaluada como indicador de procesos normales o patológicos, o una respuesta farmacológica a una intervención terapéutica. Puede ser una sustancia bioquímica a partir de una muestra biológica o un registro (presión arterial, electrocardiograma, Holter) o un examen de imagen (ecocardiografía, tomografía computarizada).

Es indicador de factor de riesgo, estado (preclínica/clínica) o tasa de enfermedad (progresión) convirtiéndose en una característica que se puede medir y evaluar objetivamente ${ }^{5}$.

\section{Clasificación de los biomarcadores}

Los biomarcadores de interés en la IC pueden agruparse de forma general, según el conocimiento actual de su papel en la fisiopatología del trastorno subyacente (Tabla 1 ).

\section{Marcadores neurohormonales}

Los utilizados en la práctica clínica habitual son los péptidos natriuréticos $(\mathrm{PN})$ tipo $\mathrm{B}$ (BNP y NTproBNP $)^{2}$. Los $\mathrm{PN}$ facilitan el filtrado glomerular y la excreción de sodio, inhiben la vasoconstricción/ retención de sodio del sistema renina-angiotensina-aldosterona (SRAA) y ejercen un efecto tónico antitrófico que atenúa la fibrosis intersticial y la hipertrofia cardiaca. El estímulo secretor clave es

\section{Tabla 1. Clasificación de los biomarcadores}

A) Marcadores neurohormonales
A1) Péptidos natriuréticos cardiacos
Péptidos natriuréticos tipo B (BNP, NT-pro BNP,
pro BNP)
ANP, NT-pro ANP, pro ANP de region media
Péptidos natriuréticos de tipo C (CNP, NT-pro CNP)
A2) Sistema renina-angiotensina-aldosterona
Actividad de renina plasmática
Angiotensina II
Aldosterona
A3) Sistema nervioso adrenérgico
Noradrenalina
Adrenalina
A4) Arginina-vasopresina
AVP
Copeptina
A5) Péptidos derivados de endotelina
Endotelina 1, endotelina grande
Adrenomedulina, proadrenomedulina de región
media
Urocortinas I, II, III
B) Marcadores inflamatorios
Proteína C reactiva
Factor de necrosis tumoral alfa
Fas (APO-1)
Interleucinas 1,6 y 18

\section{C) Marcadores de estrés oxidativo}

LDL oxidadas

Mieloperoxidas

Biopirrinas urinarias

Isoprostanos urinarios y plasmáticos

Malondialdehído plasmáticos

Carbonilproteínas

D) Marcadores del remodelado de matriz intersticial

Metaloproteinasas de matriz

Inhibidores tisulares de metaloproteinasas

Propéptido procolágeno I

Procolágeno III

\section{E) Marcadores de injuria miocitaria}

\author{
Troponinas I y $T$ cardiacas \\ Creatincinasa, CK MB \\ Mioglobina \\ Cinasa de cadenas ligeras de miosina I \\ Proteínas fijadoras de ácidos grasos cardiacos \\ Albúmina modificada por isquemia
}

\section{F) Nuevos marcadores}

ST2, Adiponectina, Osteoprotegerina,

Galectina 3, Coenzima Q10,

Factor de diferenciación del crecimiento 15

NGAL (Lipocalina 2)

BNP: péptido natriurético tipo B; NT-pro BNP: fragmento N-terminal del precursor del BNP; pro BNP: precursor del BNP; ANP: péptido natriurético tipo $A$; CNP: péptido natriurético tipo C; AVP: Arginina-vasopresina u hormona antidiurética (ADH); LDL: low density lipoprotein; CK MB: creatincinasa isoenzima MB o creatine kinase-myocardial band; NGAL: lipocalina asociada a la gelatinasa de neutrófilos, también conocida como lipocalina-2(Lcn2). 
la distensión de los miocardiocitos y el aumento de las presiones intracardiacas.

El nivel de los PN se relaciona con la severidad y el pronóstico. De esta manera, valores promedios son mayores a peor clase funcional (CF), mayor deterioro de la función ventricular y peor condición clínica.

En una revisión de 19 estudios, Doust y cols. demostraron que el BNP es un potente predictor de riesgo en todas las etapas de la IC, siendo mejor marcador que la CF (NYHA), creatinina y fracción de eyección de ventrículo izquierdo (FEVI). Por cada $100 \mathrm{pg} / \mathrm{mL}$ de aumento, el riesgo de muerte aumenta $35 \%$ en pacientes con IC. En pacientes sin IC, el riesgo relativo de desarrollarla se duplica con un valor $>$ de $20 \mathrm{pg} / \mathrm{mL}^{6}$. Diversos trabajos señalan que niveles de BNP menores de 100 pg/ $\mathrm{ml}$ se asocian a baja probabilidad de diagnóstico de IC con un valor predictivo negativo de $90 \%$, y cuando son mayores de $400 \mathrm{pg} / \mathrm{ml}$ la probabilidad aumenta de manera significativa. Niveles mayores de $700 \mathrm{pg} / \mathrm{ml}$ implican requerimientos de tratamiento intensivo según algunas series ${ }^{7}$.

En el caso de NT pro-BNP los puntos de corte se establecen en $300 \mathrm{ng} / \mathrm{l}$ como límite inferior (valor predictivo negativo de $99 \%$ ) y $900 \mathrm{ng} / \mathrm{l}$ como límite superior ${ }^{8}$.

Han demostrado su utilidad en el control y ajuste terapéutico de la IC, lo que sugiere su importancia en el seguimiento de la enfermedad en hospitalizados y programas médicos ambulatorios ${ }^{9}$. El futuro de los PN se encuentra en la estratificación del riesgo en otras enfermedades cardiacas, como el síndrome coronario agudo y posiblemente, para determinar la gravedad de las enfermedades valvulares ${ }^{10,11}$.

Recientes datos de Richards y cols. indican que el ajuste del tratamiento en la IC crónica mediante la medición seriada de estos péptidos, en conjunto con métodos clínicos establecidos, es probable que reduzca la mortalidad cardiaca y el ingreso hospitalario por descompensación, por lo menos en aquellos con falla sistólica, menores de 75 años y con escaso número de comorbilidades ${ }^{12}$.

La endotelina es una sustancia producida por el endotelio vascular, y contribuye a la regulación de la función miocárdica, tono vascular y resistencia periférica. Las concentraciones plasmáticas son mayores en pacientes con IC; estudios experimentales sugieren que la endotelina se libera en parte de los miocitos cardiacos y endotelio vascular co- ronario, y que la angiotensina II puede contribuir a los altos niveles circulantes en $\mathrm{IC}^{13}$. A largo plazo, altos niveles de endotelina (como de angiotensina II) pueden ser perjudiciales a la remodelación miocárdica; esto ha llevado a la evaluación de la inhibición de la endotelina como una terapia para la falla cardiaca ${ }^{14}$. Sin embargo, los resultados no han sido favorables y la determinación de sus niveles no se realiza en la práctica clínica habitual.

Vasopresina es liberada desde la neurohipófisis en respuesta a cambios de osmolaridad, aumentando su concentración plasmática en la IC y asociándose a disfunción ventricular izquierda. Su liberación también es estimulada a través de barorreceptores atriales y arteriales en respuesta a la hipotensión o depleción de volumen. Este estímulo no osmótico es el motivo principal de la secreción de vasopresina en condiciones patológicas como la IC congestiva. Sin embargo, como tal, no es útil como marcador por su rápida degradación plasmática. Copeptina, un fragmento del precursor de la vasopresina (Pre-pro-vasopresina), no tiene este inconveniente, demostrando ser un importante predictor de mortalidad ${ }^{15}$ y correlacionándose con un aumento del riesgo de muerte por todas las causas en la cohorte de pacientes mayores con síntomas de IC estudiada por Alehagen ${ }^{16}$.

Actualmente, se investiga su utilidad en el "screening" del síndrome coronario agudo en combinación con troponinas, destacando su gran valor predictivo negativo ${ }^{17}$.

Adrenomedulina es una prohormona producida y secretada por el endotelio vascular, posee una secuencia de aminoácidos similar al péptido relacionado al gen de la calcitonina humana. Tiene efecto vasodilatador, inhibe la proliferación y migración del músculo liso, disminuyendo el estrés oxidativo $^{18}$. Mejora la contractilidad miocárdica a través de un mecanismo independiente del AMP cíclico. El fragmento regional medio de adrenomedulina es más estable y fácil de medir, demostrando su utilidad en el diagnóstico de la IC aguda en el estudio BACH ("Biomarkers in Acute Heart Failure") ${ }^{19}$ y ser un predictor independiente de mortalidad, lo cual agrega información pronóstica complementaria a otros biomarcadores como NT-proBNP20.

\section{Marcadores inflamatorios}

Proteína C reactiva (PCR) es un reactante de fase aguda sintetizado en los hepatocitos y células 
del músculo liso de las arterias ateroscleróticas en respuesta a citoquinas proinflamatorias como IL- $6^{21}$.

Desempeña un rol trascendental en la respuesta inmune, aterogénesis y vulnerabilidad de la placa. En 1956, se reportó que fue detectable en 30 de 40 pacientes estudiados con falla cardiaca crónica y que esta fue más severa en aquellos con niveles más altos de dicho marcador ${ }^{22}$.

Análisis multivariados indican que el incremento en el nivel de PCR es un predictor independiente de resultados adversos en pacientes con falla aguda o crónica ${ }^{23}$. En el Framingham Heart Study, se encontró que la PCR contribuye a identificar pacientes asintomáticos con riesgo de desarrollar IC, correlacionándose directamente con la severidad ${ }^{22,24}$.

Existe evidencia acerca de los efectos adversos sobre el endotelio vascular por reducción en la liberación de óxido nítrico e incremento en la producción de endotelina-1, así como en la expresión de moléculas de adhesión ${ }^{25}$.

La hipótesis del rol de las citoquinas en la IC propone la existencia de un evento precipitante, como por ejemplo isquemia miocárdica, gatillando una respuesta innata al estrés, que incluye la elaboración de citoquinas proinflamatorias, y que la expresión de éstas estaría asociada con efectos deletéreos sobre la función del ventrículo izquierdo y aceleraría la progresión de la falla cardiaca ${ }^{26}$.

Factor de necrosis tumoral alfa (TNF- $\alpha$ ) es una citoquina proinflamatoria producida en monocitos/macrófagos activados. Es creciente la evidencia que implica al TNF- $\alpha$ con la patogenia de la IC. Sabemos que el corazón sano no produce TNF- $\alpha$, pero sí el miocardio insuficiente, llevando a los miocitos a apoptosis/necrosis, acelerando el curso de la enfermedad ${ }^{27}$. Concentraciones elevadas tienen directa correlación con la CF en la que estos pacientes se encuentran ${ }^{28}$; asimismo, existe una relación lineal como factor pronóstico. En 1990, Levine et al describieron su aumento en la falla cardiaca; y junto a IL-6 predicen el desarrollo de síntomas en adultos mayores ${ }^{29}$. Dichas concentraciones son responsables de la disminución en la expresión de receptores miocárdicos de TNF- $\alpha$ observada en la IC.

ST-2 es un miembro de la familia de receptores IL-1, los cuales se unen a la IL-33, siendo producidos y liberados por la distensión de miocitos. Sus niveles guardan relación con la severidad de la falla de bomba ${ }^{30}$. La elevación durante un período de 2 semanas es un predictor de muerte o necesidad de trasplante cardiaco ${ }^{31}$.

Fas (Apo-1) es miembro de la familia del TNF que media la apoptosis celular. En la IC se produce un aumento de la forma soluble de Fas, ayudando a la detección de pacientes asintomáticos y en la estratificación del riesgo ${ }^{32}$. La inhibición del Fas soluble disminuye la remodelación ventricular después del infarto, mejorando la sobrevida ${ }^{33}$.

Estudios experimentales con pentoxifilina e inmunoglobulina endovenosa han demostrado disminuir los niveles plasmáticos de PCR y Fas en cardiopatía isquémica y dilatada, mejorando la función ventricular izquierda ${ }^{34}$.

\section{Marcadores de estrés oxidativo}

En la IC se produce aumento del estrés oxidativo debido a la generación de moléculas oxígeno-reactivas y disminución de antioxidantes endógenos, siendo responsables de la disfunción endotelial ${ }^{35}$ y progresión de la enfermedad.

Las especies oxígeno-reactivas deterioran la función contráctil mediante la modificación de proteínas centrales de acoplamiento excitacióncontracción ${ }^{36}$. Además, activan una amplia variedad de quinasas que median la hipertrofia y factores de transcripción que median la apoptosis celular.

Aunque no exista un marcador directo de estrés oxidativo medible en sangre, existen marcadores indirectos como los niveles plasmáticos de LDL oxidada, malondialdehído, mieloperoxidasa e isoprostano, estos dos últimos con excelente correlación con la gravedad siendo predictores independientes de mortalidad ${ }^{4,37}$. Sin embargo, su uso se encuentra limitado a estudios de investigación.

Un biomarcador indirecto simple y fácilmente disponible es el ácido úrico, como resultado de una mayor actividad de la xantino-oxidasa. Diversos estudios básicos y clínicos sugieren que participa activamente en la génesis y progresión de la IC correlacionándose con el deterioro hemodinámico y prediciendo un pronóstico adverso en estos pacientes $^{38}$ - mientras otras investigaciones postulan una acción beneficiosa del ácido úrico a través de su actividad antioxidante ${ }^{39}$.

\section{Marcadores de remodelado de matriz intersticial}

Otro de los hechos estudiados es la mayor degradación de la matriz extracelular debido a la 
disminución de los inhibidores de las metaloproteinasas, que conducen a un aumento del colágeno, dilatación ventricular y la subsecuente remodelación con los efectos deletéreos ya conocidos. Cicoira et al encontraron que el procolágeno III es un predictor independiente de mal pronóstico en IC, sugiriendo que podría ser la expresión del gran recambio intersticial ${ }^{40}$.

El estudio RALES ("Randomized Aldactone Evaluation Study") demostró que la espironolactona añadida al tratamiento recomendado de la IC por disfunción sistólica y síntomas severos (NYHA III o IV) reduce la muerte de cualquier causa y el riesgo de hospitalización por causa cardiovascular"1. El EPHESUS ("Eplerenone PostAcute Myocardial Infarction Heart Failure Efficacy and Survival") demostró que la eplerenona añadido al tratamiento médico de IC reduce la mortalidad de cualquier causa y hospitalización por causa cardiovascular en pacientes con infarto agudo al miocardio complicados con disfunción sistólica e $\mathrm{IC}^{42}$.

El EMPHASIS-HF ("Eplerenone in Mild Patients Hospitalizations and Survival Study in Heart Failure") demostró el efecto beneficioso de añadir eplerenona, al tratamiento recomendado en IC por disfunción sistólica y síntomas leves (NYHA II) ${ }^{43}$.

Estos estudios demostraron la importancia del efecto antialdosterónico en la disminución de la síntesis miocárdica de colágeno y el remodelado ventricular izquierdo posterior al evento isquémico, lo que sugiere su importancia terapéutica y fisiopatológica.

\section{Marcadores de injuria miocitaria}

Son liberados como resultado del estrés parietal, aumento de fenómenos inflamatorios, estrés oxidativo y activación neurohormonal. La elevación de troponinas ( $\mathrm{Tn}$ ) identifica individuos con IC estable o admitidos por una descompensación, con mayor incidencia de eventos.

Horwhich et al observaron que la TnI fue mayor de $0,04 \mathrm{ng} / \mathrm{ml}$ en aproximadamente la mitad de los pacientes con IC crónica avanzada sin isquemia, siendo considerado un predictor independiente de muerte posterior al ajuste multivariado ${ }^{44}$.

En IC crónica la TnT con valores mayores de $0,02 \mathrm{ng} / \mathrm{ml}$ se asoció con una razón de riesgo mayor de 4 para muerte ${ }^{45}$. En la supervivencia a largo plazo, tanto en pacientes con edema pul- monar agudo o IC descompensada sin incluir los síndromes coronarios agudos, hay una diferencia claramente marcada en los grupos con aumento en los niveles de troponina, independiente del valor de corte ${ }^{46,47}$. Este concepto ha generado un cambio en la concepción de estrategias de manejo en la IC descompensada, donde la prevención de la injuria miocítica cumple un rol crucial ${ }^{6}$.

Otros marcadores como miosina de cadena ligera (MLC-1), proteína transportadora de los ácidos grasos cardiacos (hFABP) y CK-MB también aumentan en IC y son predictores de muerte y rehospitalización ${ }^{48}$.

\section{Utilidad clínica}

Por décadas, los distintos biomarcadores se han utilizado principalmente en pacientes con cardiopatía isquémica aguda. Sin embargo, con el advenimiento de la Tn ultrasensible y la mayor disponibilidad de los PN, se ha ampliado su utilidad en la identificación de pacientes con IC ya sea aguda como crónica.

Dentro del gran número de sustancias estudiadas, es importante considerar una serie de variables que deben cumplir para su aplicabilidad clínica. Estos criterios son: a) análisis accesibles, estandarizados y de costo aceptable; b) asociación consistente de sus valores con el diagnóstico y el pronóstico en la IC, y c) facilitación o guía en el manejo terapéutico en la $\mathrm{IC}^{49}$.

Muchos de estos marcadores brindan información respecto a la patogénesis de la IC, además de permitir la identificación de sujetos en riesgo o la estratificación de los mismos, siendo un complemento para el diagnóstico y monitorización de la terapia médica (Tabla 2).

Recientemente, Januzzi et al demostraron la utilidad clínica de las determinaciones seriadas de NT-proBNP para monitorizar el tratamiento ambulatorio de los pacientes con IC y fracción de eyección disminuida. Según este estudio, la terapia guiada mediante NT-proBNP se asoció a una reducción de la incidencia de eventos clínicos adversos, mejoría en los parámetros de calidad de vida y efecto beneficioso sobre el remodelado ventricular?.

Importante es la influencia de la función renal sobre los niveles de estos biomarcadores. El NTproBNP es eliminado principalmente vía renal, 
Tabla 2. Resumen principales características de los biomarcadores en IC

\begin{tabular}{|c|c|c|c|c|}
\hline Biomarcador & Marcador & Fisiopatología & Rol potencial & $\begin{array}{c}\text { Guía de } \\
\text { tratamiento }\end{array}$ \\
\hline \multirow[t]{2}{*}{$\begin{array}{l}\text { BNP y } \\
\text { NT-proBNP }\end{array}$} & \multirow[t]{2}{*}{ Neurohormonal } & $\begin{array}{l}\text { Se sintetizan y liberan predomi- } \\
\text { nantemente en el miocardio ven- } \\
\text { tricular en respuesta a dilatación y } \\
\text { sobrecarga de presión }\end{array}$ & $\begin{array}{l}\text { Niveles séricos guardan directa } \\
\text { relación al grado de disfunción } \\
\text { ventricular y a severidad de su } \\
\text { sintomatología }\end{array}$ & \multirow[t]{2}{*}{+++} \\
\hline & & $\begin{array}{l}\text { Funciones incluyen vasodilatación, } \\
\text { natriuresis e inhibición del sistema } \\
\text { nervioso simpático y del eje RAA }\end{array}$ & $\begin{array}{l}\text { En pacientes con fracción de } \\
\text { eyección deprimida son buenos } \\
\text { marcadores pronósticos, algo que } \\
\text { no está tan claro en aquellos con } \\
\text { función ventricular conservada }\end{array}$ & \\
\hline \multirow[t]{2}{*}{ Copeptina } & \multirow[t]{2}{*}{ Neurohormonal } & $\begin{array}{l}\text { Fragmento del precursor de la } \\
\text { Vasopresina }\end{array}$ & $\begin{array}{l}\text { Rol independiente como marca- } \\
\text { dor pronóstico }\end{array}$ & \multirow[t]{2}{*}{++} \\
\hline & & $\begin{array}{l}\text { Niveles elevados de ADH contribu- } \\
\text { yen al desarrollo de hiponatremia, } \\
\text { vasoconstricción y remodelado } \\
\text { cardiaco adverso }\end{array}$ & $\begin{array}{l}\text { Rol potencial como marcador } \\
\text { molecular para terapias con anta- } \\
\text { gonistas de vasopresina }\end{array}$ & \\
\hline \multirow[t]{2}{*}{$\begin{array}{l}\text { Fragmento } \\
\text { medio ADM }\end{array}$} & \multirow[t]{2}{*}{ Neurohormonal } & $\begin{array}{l}\text { Producido en respuesta a estrés } \\
\text { hemodinámico }\end{array}$ & \multirow{2}{*}{$\begin{array}{l}\text { Valor pronóstico agregado } \\
\text { en combinación con péptidos } \\
\text { natriuréticos y factores de riesgo } \\
\text { clínicos tradicionales }\end{array}$} & \multirow[t]{2}{*}{+} \\
\hline & & $\begin{array}{l}\text { Efectos inótropo positivo y vaso- } \\
\text { dilatador }\end{array}$ & & \\
\hline $\begin{array}{l}\text { PCR } \\
\text { ultrasensible }\end{array}$ & Inflamatorio & $\begin{array}{l}\text { Reactante de fase aguda sinteti- } \\
\text { zado en respuesta a citoquinas } \\
\text { proinflamatorias }\end{array}$ & $\begin{array}{l}\text { Incremento en el nivel de PCR es } \\
\text { un predictor independiente de } \\
\text { resultados adversos en pacientes } \\
\text { con falla aguda o crónica }\end{array}$ & + \\
\hline \multirow[t]{3}{*}{$\begin{array}{l}\text { Troponinas } \\
(T \text { e I) }\end{array}$} & \multirow[t]{3}{*}{$\begin{array}{l}\text { Injuria } \\
\text { miocitaria }\end{array}$} & $\begin{array}{l}\text { Proteínas involucradas en la } \\
\text { contracción muscular (cardiaca y } \\
\text { esquelética) }\end{array}$ & $\begin{array}{l}\text { Predictor de mortalidad en } \\
\text { pacientes IC sin enfermedad } \\
\text { coronaria }\end{array}$ & \multirow[t]{3}{*}{ Desconocido } \\
\hline & & \multirow{2}{*}{$\begin{array}{l}\text { Liberación posterior a la pér- } \\
\text { dida de miocitos por necrosis, } \\
\text { apoptosis o injuria reversible con } \\
\text { incremento en la permeabilidad } \\
\text { de la membrana }\end{array}$} & $\begin{array}{l}\text { Predictor independiente de morbi- } \\
\text { mortalidad }\end{array}$ & \\
\hline & & & $\begin{array}{l}\text { Efecto pronóstico de los cambios } \\
\text { de niveles plasmáticos en contro- } \\
\text { les seriados es desconocido }\end{array}$ & \\
\hline \multirow[t]{2}{*}{ ST2 } & \multirow[t]{2}{*}{ Inflamatorio } & $\begin{array}{l}\text { Producidos y liberados por la } \\
\text { distensión de miocitos }\end{array}$ & \multirow[t]{2}{*}{$\begin{array}{l}\text { Predictor independiente de morbi- } \\
\text { mortalidad }\end{array}$} & \multirow[t]{2}{*}{+} \\
\hline & & $\begin{array}{l}\text { Posible mediador de la hipertrofia } \\
\text { y fibrosis miocárdica }\end{array}$ & & \\
\hline \multirow[t]{3}{*}{ NGAL } & \multirow[t]{3}{*}{$\begin{array}{l}\text { Inflamación e } \\
\text { injuria }\end{array}$} & \multirow[t]{3}{*}{$\begin{array}{l}\text { Marcador precoz de daño tubular } \\
\text { renal agudo }\end{array}$} & $\begin{array}{l}\text { Detección precoz de daño renal } \\
\text { en pacientes con IC crónica. }\end{array}$ & \multirow[t]{3}{*}{++} \\
\hline & & & $\begin{array}{l}\text { Marcador de Sd. cardio-renal más } \\
\text { efectivo que la creatininemia. }\end{array}$ & \\
\hline & & & $\begin{array}{l}\text { Predictor independiente de } \\
\text { mortalidad }\end{array}$ & \\
\hline
\end{tabular}

ADH: hormona antidiurética o Vasopresina; ADM: Adrenomedulina; BNP: péptido natriurético tipo B; NGAL: lipocalina asociada a la gelatinasa de neutrófilos; NT-pro BNP: fragmento N-terminal del precursor del BNP; PCR: proteína C reactiva; RAA: reninaangiotensina-aldosterona. 
mientras el BNP principalmente por endopeptidasas y aclaramiento por parte de receptores específicos. Esto implica que la disfunción renal adquiere mayor relevancia en el NT-proBNP que sobre $\mathrm{BNP}^{50}$.

Dentro de los biomarcadores más recientes y que ha generado mayor interés, se encuentra la copeptina, la que demostró ser un potente predictor de mortalidad en pacientes con IC, especialmente aquellos con síntomas leves o moderados, que son más difíciles de evaluar en forma ambulatoria, en el trabajo de Neuhold et $\mathrm{a}^{51}$; lo que la transforma en una nueva herramienta de trabajo ${ }^{52}$, con la idea de identificar a los pacientes que se podrían beneficiar de un tratamiento con drogas antagonistas de los receptores renales de la vasopresina.

Los péptidos de tipo $\mathrm{B}$ son los únicos biomarcadores realmente establecidos en la práctica clínica en la $\mathrm{IC}^{53}$. Actualmente, se recomienda su utilización para excluir otras alternativas como causa de disnea y para obtener información pronóstica (Recomendación Clase IIa - Nivel de Evidencia C $)^{54}$. Debe tenerse en cuenta que la afección de sus niveles guardan relación con la edad, sexo, hábito corporal, función renal y tiroidea, anemia, alteraciones del ritmo y cirugía cardiaca reciente. También se ve aumento de sus niveles con el tratamiento a largo plazo con inhibidores de la enzima convertidora de angiotensina, bloqueadores de los receptores de angiotensina y espironolactona ${ }^{55}$. Los niveles pueden ser inicialmente normales en las etapas agudas de la IC e insuficiencia mitral.

Bettencourt et al observaron que la falta de normalización en los niveles de BNP durante la hospitalización predecía mayor riesgo de muerte y rehospitalización, con una mayor sobrevida libre de eventos con niveles inferiores a $250 \mathrm{pg} / \mathrm{ml}$ al momento del alta ${ }^{56}$.

A modo de resumen, para los pacientes que presentan aparición aguda de síntomas o empeoramiento de estos, el punto de corte óptimo de exclusión es de 300 pg/mL para NT-proBNP y 100 $\mathrm{pg} / \mathrm{ml}$ para BNP. Para aquellos que se presentan en una forma no aguda, el punto de corte es de $125 \mathrm{pg} / \mathrm{mL}$ para el NT-proBNP y $35 \mathrm{pg} \mathrm{ml} /$ para BNP. La sensibilidad y especificidad de BNP y NTproBNP para el diagnóstico de IC son más bajos en pacientes no agudos ${ }^{54}$.

El tratamiento de la IC guiado por biomarcadores parece mejorar la calidad de vida de los pacientes, particularmente en aquellos que logran reducciones sustanciales en los niveles de $\mathrm{PN}^{57}$. En pacientes con fracción de eyección deprimida son buenos marcadores pronósticos, algo que no está tan claro en pacientes con insuficiencia cardiaca con función ventricular conservada ${ }^{58}$.

El papel etiológico clave del SRAA y nervioso simpático en la progresión de la IC son claros, pero no se ha observado beneficio alguno con la determinación sistemática de renina, angiotensina II, aldosterona o catecolaminas plasmáticas para el diagnóstico, introducción del tratamiento o seguimiento de la IC 5 .

La combinación de dos o más biomarcadores circulantes que reflejan aspectos diferentes de la fisiopatología de la IC, tienen relación independiente con el resultado clínico, y pueden mejorar la capacidad pronóstica ${ }^{16,59}$. A modo de ejemplos, el fragmento medio de la pro-adrenomedulina agrega información pronóstica al NT-proBNP ${ }^{20}$. Januzzi et al estudiaron las concentraciones de NT-proBNP y ST2 en pacientes con IC aguda que acudieron a Urgencias observando que la elevación simultánea de ambos biomarcadores comportaba un riesgo de mortalidad muy superior al asociado a la elevación de uno solo de ellos ${ }^{60}$.

Hay marcadores emergentes en estudio que aportarán información pronóstica y mejorarán la estratificación de riesgo en IC.

\section{Referencias}

1. Thom T, Haase N, Rosamond W, Howard VJ, Rumsfeld J, Manolio T, et al. Heart disease and stroke statistics-2006 update: a report from the American Heart Association Statistics Committee and Stroke Statistics Subcommittee. Circulation 2006; 113 (6): e85-151. Epub 2006/01/13.

2. Chen WC, Tran KD, Maisel AS. Biomarkers in heart failure. Heart 2010; 96 (4): 314-20. Epub 2010/03/03.

3. Mueller C, Scholer A, Laule-Kilian K, Martina B, Schindler C, Buser P, et al. Use of B-type natriuretic peptide in the evaluation and management of acute dyspnea. $\mathrm{N}$ Engl J Med 2004; 350 (7): 647-54. Epub 2004/02/13.

4. Miranda HR, Castro GP, Verdejo PH, Chiong M, DíazAraya G, Mellado R, et al. [Oxidative stress and inflammation in heart failure: mechanisms of damage and therapeutic alternatives]. Rev Med Chile 2007; 135 (8): 1056-63. Epub 2007/11/09. Estrés oxidativo e inflamación en insuficiencia cardiaca: Mecanismos de daño y alternativas terapéuticas. 
5. Richards AM. New biomarkers in heart failure: applications in diagnosis, prognosis and guidance of therapy. Rev Esp Cardiol 2010; 63 (6): 635-9. Epub 2010/06/03.

6. Doust JA, Pietrzak E, Dobson A, Glasziou P. How well does B-type natriuretic peptide predict death and cardiac events in patients with heart failure: systematic review. BMJ 2005; 330 (7492): 625. Epub 2005/03/19.

7. Logeart D, Thabut G, Jourdain P, Chavelas C, Beyne P, Beauvais F, et al. Predischarge B-type natriuretic peptide assay for identifying patients at high risk of re-admission after decompensated heart failure. J Am Coll Cardiol 2004; 43 (4): 635-41. Epub 2004/02/21.

8. Januzzi JL Jr, Camargo CA, Anwaruddin S, Baggish AL, Chen AA, Krauser DG, et al. The N-terminal Pro-BNP investigation of dyspnea in the emergency department (PRIDE) study. Am J Cardiol 2005; 95 (8): 948-54. Epub 2005/04/12.

9. Januzzi JL Jr, Rehman SU, Mohammed AA, Bhardwaj A, Barajas L, Barajas J, et al. Use of amino-terminal proB-type natriuretic peptide to guide outpatient therapy of patients with chronic left ventricular systolic dysfunction. J Am Coll Cardiol 2011;58(18):1881-9. Epub 2011/10/25.

10. Gopal DJ, Iqbal MN, Maisel A. Updating the role of natriuretic peptide levels in cardiovascular disease. Postgrad Med 2011; 123 (6): 102-13. Epub 2011/11/23.

11. Khan SQ, Quinn P, Davies JE, Ng LL. N-terminal pro-Btype natriuretic peptide is better than TIMI risk score at predicting death after acute myocardial infarction. Heart 2008; 94 (1): 40-3. Epub 2007/05/10.

12. Richards AM, Troughton RW. Use of Natriuretic Peptides to Guide and Monitor Heart Failure Therapy. Clin Chem 2011. Epub 2011/11/17.

13. Teerlink JR. Endothelins: pathophysiology and treatment implications in chronic heart failure. Curr Heart Fail Rep 2005; 2 (4): 191-7. Epub 2005/12/08.

14. Rich S, McLaughlin VV. Endothelin receptor blockers in cardiovascular disease. Circulation 2003; 108 (18): 2184-90. Epub 2003/11/05.

15. Morgenthaler NG. Copeptin: a biomarker of cardiovascular and renal function. Congest Heart Fail 2010; 16 Suppl 1: S37-44. Epub 2010/08/07.

16. Alehagen U, Dahlstrom U, Rehfeld JF, Goetze JP. Association of copeptin and $\mathrm{N}$-terminal proBNP concentrations with risk of cardiovascular death in older patients with symptoms of heart failure. JAMA 2011; 305 (20): 2088 95. Epub 2011/05/26.

17. Keller T, Tzikas S, Zeller T, Czyz E, Lillpopp L, Ojeda FM, et al. Copeptin improves early diagnosis of acute myocardial infarction. J Am Coll Cardiol 2010; 55 (19): 2096-106. Epub 2010/05/08.
18. Kohno M, Yokokawa K, Kano H, Yasunari K, Minami M, Hanehira T, et al. Adrenomedullin is a potent inhibitor of angiotensin II-induced migration of human coronary artery smooth muscle cells. Hypertension 1997; 29 (6): 1309-13. Epub 1997/06/01.

19. Maisel A, Mueller C, Nowak R, Peacock WF, Landsberg JW, Ponikowski P, et al. Mid-region pro-hormone markers for diagnosis and prognosis in acute dyspnea: results from the BACH (Biomarkers in Acute Heart Failure) trial. J Am Coll Cardiol 2010; 55 (19): 2062-76. Epub 2010/05/08.

20. von Haehling S, Filippatos GS, Papassotiriou J, Cicoira M, Jankowska EA, Doehner W, et al. Mid-regional proadrenomedullin as a novel predictor of mortality in patients with chronic heart failure. Eur J Heart Fail 2010; 12 (5): 484-91. Epub 2010/03/11.

21. Calabro P, Willerson JT, Yeh ET. Inflammatory cytokines stimulated C-reactive protein production by human coronary artery smooth muscle cells. Circulation 2003; 108 (16): 1930-2. Epub 2003/10/08.

22. Elster SK, Braunwald E, Wood HF. A study of C-reactive protein in the serum of patients with congestive heart failure. Am Heart J 1956; 51 (4): 533-41. Epub 1956/04/01.

23. Anand IS, Latini R, Florea VG, Kuskowski MA, Rector $\mathrm{T}$, Masson S, et al. C-reactive protein in heart failure: prognostic value and the effect of valsartan. Circulation 2005; 112 (10): 1428-34. Epub 2005/09/01.

24. Vasan RS, Sullivan LM, Roubenoff R, Dinarello CA, Harris T, Benjamin EJ, et al. Inflammatory markers and risk of heart failure in elderly subjects without prior myocardial infarction: the Framingham Heart Study. Circulation 2003; 107 (11): 1486-91. Epub 2003/03/26.

25. Venugopal SK, Devaraj S, Jialal I. Effect of C-reactive protein on vascular cells: evidence for a proinflammatory, proatherogenic role. Curr Opin Nephrol Hypertens 2005; 14 (1): 33-7. Epub 2004/12/09.

26. Seta Y, Shan K, Bozkurt B, Oral H, Mann DL. Basic mechanisms in heart failure: the cytokine hypothesis. J Card Fail 1996; 2 (3): 243-9. Epub 1996/09/01.

27. Anker SD, von Haehling S. Inflammatory mediators in chronic heart failure: an overview. Heart 2004; 90 (4): 464-70. Epub 2004/03/17.

28. Herrera-Garza EH, Stetson SJ, Cubillos-Garzon A, Vooletich MT, Farmer JA, Torre-Amione G. Tumor necrosis factor-alpha: a mediator of disease progression in the failing human heart. Chest 1999; 115 (4): 1170-4. Epub 1999/04/20.

29. Lee DS, Vasan RS. Novel markers for heart failure diagnosis and prognosis. Curr Opin Cardiol 2005; 20 (3): 201-10. Epub 2005/04/30. 
30. Bayes-Genis A, Pascual-Figal D, Januzzi JL, Maisel A, Casas T, Valdes Chavarri M, et al. Soluble ST2 monitoring provides additional risk stratification for outpatients with decompensated heart failure. Rev Esp Cardiol 2010; 63 (10): 1171-8. Epub 2010/09/30.

31. Weinberg EO, Shimpo M, Hurwitz S, Tominaga S, Rouleau JL, Lee RT. Identification of serum soluble ST2 receptor as a novel heart failure biomarker. Circulation 2003; 107 (5): 721-6. Epub 2003/02/13.

32. Okuyama M, Yamaguchi S, Nozaki N, Yamaoka M, Shirakabe $\mathrm{M}$, Tomoike H. Serum levels of soluble form of Fas molecule in patients with congestive heart failure. Am J Cardiol 1997; 79 (12): 1698-701. Epub 1997/06/15.

33. Li Y, Takemura G, Kosai K, Takahashi T, Okada H, Miyata $\mathrm{S}$, et al. Critical roles for the Fas/Fas ligand system in postinfarction ventricular remodeling and heart failure. Circ Res 2004; 95 (6): 627-36. Epub 2004/08/07.

34. Gullestad L, Aukrust P. Review of trials in chronic heart failure showing broad-spectrum anti-inflammatory approaches. Am J Cardiol 2005; 95 (11A): 17C-23C; discussion 38C-40C. Epub 2005/06/01.

35. Greig D, Castro P, Gabrielli L, Miranda R, Verdejo H, Alcaino $\mathrm{H}$, et al. [Inflammation and endothelial dysfunction in patients with chronic heart failure]. Rev Med Chile 2008; 136 (6): 687-93. Epub 2008/09/05. Inflamacion y disfuncion endotelial en pacientes con insuficiencia cardiaca crónica.

36. Tsutsui H, Kinugawa S, Matsushima S. Oxidative stress and heart failure. Am J Physiol Heart Circul Physiol 2011; 301 (6): H2181-90. Epub 2011/09/29.

37. Kameda K, Matsunaga T, Abe N, Hanada H, Ishizaka $\mathrm{H}$, Ono $\mathrm{H}$, et al. Correlation of oxidative stress with activity of matrix metalloproteinase in patients with coronary artery disease. Possible role for left ventricular remodelling. Eur Heart J 2003; 24 (24): 2180-5. Epub 2003/12/09

38. Feig DI, Kang DH, Johnson RJ. Uric acid and cardiovascular risk. N Engl J Med 2008; 359 (17): 1811-21. Epub 2008/10/24.

39. Alcaino H, Greig D, Castro P, Verdejo H, Mellado R, García L, et al. [The role of uric acid in heart failure]. Rev Med Chile 2011; 139 (4): 505-15. Epub 2011/09/01. Ácido úrico: una molécula con acciones paradójicas en la insuficiencia cardiaca.

40. Cicoira M, Rossi A, Bonapace S, Zanolla L, Golia G, Franceschini L, et al. Independent and additional prognostic value of aminoterminal propeptide of type III procollagen circulating levels in patients with chronic heart failure. J Card Fail 2004; 10 (5): 403-11. Epub 2004/10/08

41. Zannad F, Alla F, Dousset B, Pérez A, Pitt B. Limitation of excessive extracellular matrix turnover may contribute to survival benefit of spironolactone therapy in patients with congestive heart failure: insights from the randomized aldactone evaluation study (RALES). Rales Investigators. Circulation 2000; 102 (22): 2700-6. Epub 2000/11/30

42. Pitt B, Remme W, Zannad F, Neaton J, Martínez F, Roniker B, et al. Eplerenone, a selective aldosterone blocker, in patients with left ventricular dysfunction after myocardial infarction. N Engl J Med 2003; 348 (14): 1309-21. Epub 2003/04/02.

43. Zannad F, McMurray JJ, Krum H, van Veldhuisen DJ, Swedberg K, Shi H, et al. Eplerenone in patients with systolic heart failure and mild symptoms. N Engl J Med 2011; 364 (1): 11-21. Epub 2010/11/16.

44. Horwich TB, Patel J, MacLellan WR, Fonarow GC. Cardiac troponin I is associated with impaired hemodynamics, progressive left ventricular dysfunction, and increased mortality rates in advanced heart failure. Circulation 2003; 108 (7): 833-8. Epub 2003/08/13.

45. Hudson MP, O'Connor CM, Gattis WA, Tasissa G, Hasselblad V, Holleman CM, et al. Implications of elevated cardiac troponin $\mathrm{T}$ in ambulatory patients with heart failure: a prospective analysis. Am Heart J 2004; 147 (3): 546-52. Epub 2004/03/05.

46. Kociol RD, Pang PS, Gheorghiade M, Fonarow GC, O'Connor CM, Felker GM. Troponin elevation in heart failure prevalence, mechanisms, and clinical implications. J Am Coll Cardiol 2010; 56 (14): 1071-8. Epub 2010/09/25.

47. Xue Y, Clopton P, Peacock WF, Maisel AS. Serial changes in high-sensitive troponin I predict outcome in patients with decompensated heart failure. Eur J Heart Fail 2011; 13 (1): 37-42. Epub 2010/12/15

48. Sugiura T, Takase H, Toriyama T, Goto T, Ueda R, Dohi Y. Circulating levels of myocardial proteins predict future deterioration of congestive heart failure. J Card Fail 2005; 11 (7): 504-9. Epub 2005/10/04.

49. Richards AM. What we may expect from biomarkers in heart failure. Heart failure clinics. 2009; 5 (4): 463-70. Epub 2009/07/28.

50. Hogenhuis J, Voors AA, Jaarsma T, Hoes AW, Hillege HL, Kragten JA, et al. Anaemia and renal dysfunction are independently associated with BNP and NT-proBNP levels in patients with heart failure. Eur J Heart Fail 2007; 9 (8): 787-94. Epub 2007/05/29.

51. Neuhold S, Huelsmann M, Strunk G, Stoiser B, Struck J, Morgenthaler NG, et al. Comparison of copeptin, B-type natriuretic peptide, and amino-terminal pro-B-type natriuretic peptide in patients with chronic heart failure: prediction of death at different stages of the disease. J 
Am Coll Cardiol 2008; 52 (4): 266-72. Epub 2008/07/19.

52. Stoiser B, Mortl D, Hulsmann M, Berger R, Struck J, Morgenthaler NG, et al. Copeptin, a fragment of the vasopressin precursor, as a novel predictor of outcome in heart failure. Eur J Clin Invest 2006; 36 (11): 771-8. Epub 2006/10/13.

53. Braunwald E. Biomarkers in heart failure. $\mathrm{N}$ Engl J Med 2008; 358 (20): 2148-59. Epub 2008/05/16.

54. McMurray JJ, Adamopoulos S, Anker SD, Auricchio A, Bohm M, Dickstein K, et al. ESC guidelines for the diagnosis and treatment of acute and chronic heart failure 2012: The Task Force for the Diagnosis and Treatment of Acute and Chronic Heart Failure 2012 of the European Society of Cardiology. Developed in collaboration with the Heart Failure Association (HFA) of the ESC. Eur J Heart Fail 2012; 14 (8): 803-69. Epub 2012/07/26.

55. Latini R, Masson S, Anand I, Salio M, Hester A, Judd $\mathrm{D}$, et al. The comparative prognostic value of plasma neurohormones at baseline in patients with heart failure enrolled in Val-HeFT. Eur Heart J 2004; 25 (4): 292-9. Epub 2004/02/27.

56. Bettencourt P, Azevedo A, Pimenta J, Frioes F, Ferreira S, Ferreira A. N-terminal-pro-brain natriuretic peptide predicts outcome after hospital discharge in heart failure patients. Circulation 2004; 110 (15): 2168-74. Epub 2004/09/29.

57. Deberadinis B, Januzzi JL Jr. Use of biomarkers to guide outpatient therapy of heart failure. Curr Opin Cardiol 2012; 27 (6): 661-8. Epub 2012/09/04.

58. Grewal J, McKelvie RS, Persson H, Tait P, Carlsson J, Swedberg K, et al. Usefulness of N-terminal pro-brain natriuretic Peptide and brain natriuretic peptide to predict cardiovascular outcomes in patients with heart failure and preserved left ventricular ejection fraction. Am J Cardiol 2008; 102 (6): 733-7. Epub 2008/09/09.

59. Nishio Y, Sato Y, Taniguchi R, Shizuta S, Doi T, Morimoto $\mathrm{T}$, et al. Cardiac troponin $\mathrm{T}$ vs other biochemical markers in patients with congestive heart failure. Circ J 2007; 71 (5): 631-5. Epub 2007/04/26.

60. Januzzi JL Jr, Peacock WF, Maisel AS, Chae CU, Jesse RL, Baggish AL, et al. Measurement of the interleukin family member ST2 in patients with acute dyspnea: results from the PRIDE (Pro-Brain Natriuretic Peptide Investigation of Dyspnea in the Emergency Department) study. J Am Coll Cardiol 2007; 50 (7): 607-13. Epub 2007/08/19. 